\title{
The impacts of maternal stress on worker phenotypes in the honey bee
}

\author{
Sarah R. Preston ${ }^{1}$, Joseph H. Palmer ${ }^{1,2}$, James W. Harrison ${ }^{1}$, Hanna M. Carr ${ }^{1}$, \\ Clare C. RitTsChOF ${ }^{1}$ \\ ${ }^{1}$ Department of Entomology, College of Agriculture, Food, and Environment, University of Kentucky, S-225 North \\ Agricultural Science Center, Lexington, KY 40546, USA \\ ${ }^{2}$ College of Agriculture, Communities, and the Environment, Kentucky State University, 400 E. Main St., Frankfort, KY \\ 40601, USA
}

Received 21 March 2019 - Revised 18 June 2019 - Accepted 15 July 2019

\begin{abstract}
Maternal stress is a common source of heritable health and behavioral variation. This type of maternal effect could be particularly important for honey bees (Apis mellifera), as a single queen is responsible for many generations of workers who perform all colony functions including raising subsequent worker generations. Multiple factors work synergistically to cause colony loss, but a role for maternal stress effects is unstudied. We used an artificial cold temperature treatment as a proof-of-concept approach to investigate whether acute queen stress causes changes in worker phenotypes, including egg hatching rate, development time, and adult behavior and immune function. We found that queen stress impacts early-life phenotypes (egg hatching and development time), with more limited impacts on adult phenotypes (behavior and immune function). Thus, if maternal stress impacts colony health, it is likely through cumulative impacts on worker population numbers, not through phenotypic effects that impact individual adult worker behavior or health resilience. This study addresses an important and overlooked question, and provides a baseline understanding of the likely impacts of queen stress on worker phenotypes.
\end{abstract}

\section{maternal effects / climate change / queen failure / immune function / gene expression}

\section{INTRODUCTION}

Maternal effects occur when a female's phenotype, genotype, or experience impact the phenotype of her offspring beyond direct genetic effects (Marshall and Uller 2007). This phenomenon is phylogenetically widespread, and can contribute greatly to offspring fitness (Räsänen and Kruuk 2007; Wolf and Wade 2009). Maternal effects can

Electronic supplementary material The online version of this article (https://doi.org/10.1007/s13592-019-00680-1) contains supplementary material, which is available to authorized users.

Corresponding author: C. Rittschof, clare.rittschof@uky.edu

Manuscript editor: Klaus Hartfelder be a form of adaptive phenotypic plasticity; when maternal experiences are communicated to offspring, it may allow them to alter aspects of their phenotype and maximize survivorship and reproductive success in spatially or temporally variable environments (Gibbs et al. 2010; Keiser and Mondor 2013; Lacour et al. 2014). Alternatively, maternal effects can reflect offspring responses to maternal stressors without clear adaptive value (Räsänen and Kruuk 2007). Specifically, shortterm maternal stressors that negatively impact the fitness of both mother and offspring are called transmissive maternal effects (Marshall and Uller 2007). Although the sources of these stressors vary, for many species, anthropogenic changes to the environment in the form of chemical, noise, and light pollution, climate change, and increased 
agricultural intensification, provide myriad stressors that impact organisms over rapid ecological timescales (Bonduriansky et al. 2012; Manley et al. 2015; Sulmon et al. 2015; Lister and Garcia 2018). Such circumstances may increase the prevalence of transmissive maternal effects, and for species in disturbed environments, such maternal stress effects could be important contributors to population declines (Crino et al. 2013; Strasser and Heath 2013; Forcada and Hoffman 2014; Jeffs and Leather 2014).

Worldwide populations of the western honey bee (Apis mellifera) have been steadily declining for the last 70 years due to synergistic impacts of a variety of anthropogenic disturbances. These include hive cultivation outside of the honey bee's native range, increased pesticide use, introduction of congeneric parasites and novel pathogens to which honey bees are not co-adapted, and loss of floral resources due to increased urban and agricultural land development (Smith et al. 2013; Goulson et al. 2015; McMenamin and Genersch 2015). Honey bee colonies are made up of 20,000-60,000 female worker bees that are all descended from a single mother (the queen) who lays thousands of eggs a day over her 34-year lifespan (Amiri et al. 2017). Despite her highly significant role in colony stability and health, and despite recent evidence of high rates of queen failure and decreased longevity in managed colonies (Tarpy et al. 2012; Pettis et al. 2016; Amiri et al. 2017), the vast majority of studies that have addressed how stressors give rise to honey bee colony mortality focus exclusively on direct impacts on worker bees (Barron 2015; Johnson 2015; Klein et al. 2017). A smaller but growing body of work is addressing how stressors impact the queen herself despite the fact that she rarely leaves the safety of the hive (Alaux et al. 2011; DeGrandi-Hoffman et al. 2013; Dussaubat et al. 2016; Amiri et al. 2017). No studies, however, have addressed how stress to the queen impacts critical developmental, behavioral, and immune phenotypes of worker bees by way of maternal effects.

Here, we evaluate whether honey bee queen stress results in transmissive maternal effects that impact worker bee offspring survivorship, development, immune function, and behavior. Sublethal impacts on worker behavior are particularly important to consider because workers perform all of the tasks required for colony function, including nursing and feeding the next generation of work bees (Winston 1987). Thus, negative impacts on even a single day's cohort of workers could have lasting effects on a colony (Khoury et al. 2011). Previous studies show that aspects of a queen's phenotype do influence worker offspring, suggesting certain types of maternal effects are possible. For example, maternal age is negatively correlated with embryo size, embryo viability, and early larval development (Al-Lawati and Bienefeld 2009). However, there is also evidence that negative environmental effects and individual variation during early development are ameliorated by the time individuals reach adulthood (Fox and Dingle 1994), which may counterbalance maternal stress effects.

In the present study, we evaluate the potential for transmissive maternal effects in honey bees by treating queens with an artificial cold temperature stress, and assessing worker phenotypes before and after queen stress. Because the effect of queen stress on offspring has not been examined, we use the artificial cold stress as a proof-of-concept approach to provide a baseline understanding of the likely impacts of queen stress on worker phenotypes. We chose this particular artificial stressor because previous studies have shown that it has strong impacts on aspects of the queen's reproductive biology (Pettis et al. 2016), making it a good candidate for a first evaluation of the presence of transmissive maternal effects. We assess how queen stress impacts variation in worker egg content, hatching success rate, development time, adult behavior, and adult immune function.

\section{MATERIALS AND METHODS}

\subsection{Overview}

We used a repeated measures design to compare a queen's offspring before and after she experienced a $2-\mathrm{h}, 4{ }^{\circ} \mathrm{C}$ cold stress treatment (Pettis et al. 2016). We chose this serial design, as opposed to a randomized treatment order, 
because the cold stress has lasting impacts on queen reproductive function (Pettis et al. 2016), and it was not clear that collecting control data after cold stress would be free of stress effects. Our design involved comparing treatments for a given queen over a relatively short time period of 3 days (see Sections 2.3 and 2.4), which minimizes potential impacts of natural seasonal changes on a queen's reproductive output. The cold exposure we used is a common stressor experienced by commercially purchased honey bee queens, which are shipped across the country by air. We selected this paradigm because it is easy to apply consistently across individuals, and it has a proven biological impact, decreased sperm viability (Pettis et al. 2016). In addition, because it is a stronger stressor than would be routinely encountered by honey bee queens, it provides a robust proof-of-concept study of the likely impacts of queen stress on worker phenotypes.

\subsection{Honey bee sources}

All honey bee colonies used in this study were of mixed genotypic origin, advertised as A.m. carnica and A.m. ligustica, purchased from local commercial beekeepers. To control for variation in queen age, quality, reproductive experience, and genotype, we purchased mated, outbred queens of uniform age without any laying experience from a single supplier. We purchased queens in blocks at the same time prior to initiating each block of the experiment (see Section 2.3, 20 total queens, Guthries Naturals, Frankfort, KY, USA). This ensured that all queens throughout the study were of the exact same age and reproductive experience at the initiation of the experiment. Experimental and foster hives (see Section 2.3) were full-sized hives (composed of 2, 10-frame, 24.45$\mathrm{cm}$-deep boxes) at the start of the experiment. Worker bees build honey comb against wooden frames containing a plastic foundation, and offspring are laid and reared inside individual honeycomb cells. Hives were maintained following traditional beekeeping standards and practices (Graham 2015). Practices complied with disease management recommendations set forth by the
Honey Bee Health Coalition and the Kentucky State Department of Agriculture.

\subsection{Experimental queen introduction}

To achieve the desired level of replication in this experiment, our goal was to collect offspring from 32 stressed and unstressed queens. However, because applying a strong stressor to a queen could have lasting impacts on colony health and ultimately lead to hive failure, we did not want to sacrifice 32 independent colonies over the course of this experiment. Instead, we continuously reused a set of 8 experimental hives to house our focal queens short-term while they laid eggs before and after the cold stress. To initiate the experiment, we introduced queens to each of the 8 experimental colonies one at a time in 4 blocks separated by 4 -week time intervals (April-August 2017). We introduced two queens a day at 2-day intervals within each block. This staggered start and additional measures taken to randomize behavioral assays (see Section 2.5) allowed us to perform behavioral analyses blind to the treatment identity of the offspring.

Introducing new queens into one of the 8 experimental hives required us to take steps to ensure the hives did not kill or reject the new queen at introduction. We followed a consistent protocol for introducing queens to our experimental colonies to ensure acceptance. First, we located and removed the queen heading the experimental hive (i.e., the queen present from the previous round of the experiment), and allowed the hive to remain queenless for 2 days. This allowed the hive to recognize the absence of the queen, which increases the likelihood the new queen will be accepted (Perez-Sato et al. 2015). After two queenless days, we placed the new queen within a wooden cage inside the hive. This allows workers to sense and feed the new queen, but not sting or kill her. After 4 days, we released the queen into the hive. We left the hive undisturbed for the next 14 days in order to allow the queen to begin laying eggs. Because honey bee worker eggs hatch within $72 \mathrm{~h}$ of being laid (Winston 1987), any eggs present after this 14day time frame were laid by the newly introduced queen, and so at this point we could collect the 
handling control and initiate the queen caging and offspring collection portion of the experiment (see Section 2.4). After we completed the stress paradigm and offspring collection for a given queen (see Section 2.4), she was removed and replaced with the next queen (see above).

\subsection{Experimental treatment groups and egg protein sample collection}

We assessed three different sets of offspring laid by experimental queens. First, 14 days following queen installation and prior to any additional disturbance to the queen or colony, we located a frame containing eggs that were approximately $24 \mathrm{~h}$ old. We estimated age based on the vertical orientation of the egg within the honeycomb cell (Winston 1987). These eggs were a "handling control," used to assess impacts of stress from queen handling on offspring phenotypes. We removed this frame and, within $30 \mathrm{~min}$, collected 20 eggs into microcentrifuge tubes (1.7 mL, VWR International, Radnor, PA, USA). We stored eggs at $-20{ }^{\circ} \mathrm{C}$ for later assessment of protein content (see Section 2.7). The frame containing the remaining eggs was labeled and placed within a foster hive (see below). We then located the queen and trapped her for $24 \mathrm{~h}$ against an empty frame with drawn honeycomb using a "push-in cage." This cage $(40.5 \mathrm{~cm}$ long by $19 \mathrm{~cm}$ wide by $3 \mathrm{~cm}$ tall) covers about $80 \%$ of one side of a frame. It is made of hardware cloth (\#8, Amazon.com, Seattle, WA, USA) with a piece of plastic queen excluder $(35 \mathrm{~cm}$ long by $15 \mathrm{~cm}$ wide, $0.5-\mathrm{cm}$ opening) glued into the center (Fig. S1). Through the queen excluder, workers are able to move in and out of the cage, but the queen cannot. Eggs laid on the frame within this 24-h caging period were designated "control." We collected eggs for protein assessment as above, and transferred the frame containing the remaining eggs to a foster hive (see below).

After the first 24-h caging period, we removed the queen using a queen clip catcher (Dadant and Sons Inc., Hamilton, IL, USA) and placed the queen (inside the clip) into a refrigerator at $4{ }^{\circ} \mathrm{C}$ for $2 \mathrm{~h}$ (following Pettis et al. 2016). The cold treatment resulted in a light chill coma, from which the queen was revived within minutes of removal from the refrigerator. After the cold treatment, we placed the queen, still inside the clip, back into the experimental hive. We allowed the queen $1 \mathrm{~h}$ to recover, during which workers were able to access the queen through the clip. After $1 \mathrm{~h}$, we trapped the queen for $24 \mathrm{~h}$ against a new, empty honeycomb frame. Pilot studies showed that eggs laid during this first 24 -h period following the cold treatment failed to hatch in most cases (75\% hatching failure, $N=4$ queens). This failure to hatch is likely due to higher cold susceptibility at the advanced developmental state (Mackensen 1951; Yu and Omholt 1999; Rinderer 2008; Baer et al. 2016). The pilot study showed that eggs laid within the second 24-h period following treatment were more likely to hatch $(100 \%$ hatching success, $N=4$ queens). Therefore, we trapped the queen for an additional $24 \mathrm{~h}$ against another new honeycomb frame, and designated the resulting offspring as the "cold stress" treatment. We collected eggs for protein analysis and transferred the frame to a foster hive (see below). We removed the queen from the colony to initiate the next block of the experiment.

Honeycomb frames containing eggs laid by experimental queens were transferred to one of 14 foster hives for the duration of their development. Foster hives were strong, otherwise undisturbed colonies whose populations remained stable throughout the experiment. This procedure served two purposes. First, it ensured adequate adult bees were present to rear focal offspring. The 8 experimental hives, weakened by repeated queen replacement (above), may not contain adequate nurses for rearing offspring (Amdam and Omholt 2002). Second, by housing the offspring in random hives, we could ensure that any treatment effects we observed were a function of stress to the queen, and not colony level effects that may indicate the adult worker response to a stressed queen. Frames of offspring were assigned to foster hives using a random number generator. We placed frames in the brood nest in the lower hive box, placed a queen excluder between the bottom and top box, and moved the foster colony queen to the top box to prevent egg laying on study frames (Rittschof et al. 2015; Traynor et al. 2017). Four days after we added frames to foster colonies (at which point all eggs from study frames would 
have hatched), we removed the queen excluder from the hive to allow the queen to move freely.

\subsection{Offspring sample collection and behavioral analyses}

Six days after each frame of eggs was transferred to the foster hive, we examined the frame for the presence of larvae (honey bee eggs take $72 \mathrm{~h}$ to hatch). Egg hatching success (which we found was all or none for a given frame of eggs) was recorded as a binary response (yes/no). We then left brood undisturbed until 1 day prior to adult emergence (17 days after the queen was removed from the frame). On this day, we removed the frame and placed it in a circulated air incubator kept at $33.5 \pm 0.5^{\circ} \mathrm{C}$ and constant darkness (Rittschof et al. 2015; Chaimanee et al. 2016; Walton and Toth 2016). In the morning and afternoon of each subsequent day, we checked frames for adult emergence. Once the offspring started to emerge, we recorded the number of bees that emerged from each frame each day. Because frames contained different numbers of offspring to begin with, and also showed variation in development time, we recorded emergence time for the first 120 offspring where possible, and fewer where frames did not contain as many bees (see Table SI for sample sizes per treatment and queen). We report development time on a perbee basis as the number of days between egg laying and emergence.

As adult offspring emerged, we placed them into petri dish cages $(100 \mathrm{~mm} \times 20 \mathrm{~mm}$, Thermo Scientific, Waltham, MA, USA) modified with an entrance hole. We provisioned bees with a $50 \% \mathrm{~m} /$ $\mathrm{v}$ sucrose solution in two 1.7-mL microcentrifuge tubes (VWR International, Radnor, PA, USA) with two small feeding holes drilled through the base (Li-Byarlay et al. 2014; Rittschof et al. 2015; Rittschof 2017). We labeled each dish with a random number derived from an online number generator. The number of dishes created per treatment and queen varied depending on offspring emergence success (see Table SII for samples sizes per treatment and queen). In most cases, we placed 4 bees in a dish, and in some cases where numbers were limited, 3 (Rittschof et al. 2018). We returned dishes to the incubator until bees were 7 days old, at which point we assessed aggressive behavior using the intruder assay (see Section 2.6, Rittschof and Robinson 2013; LiByarlay et al. 2014; Rittschof et al. 2015). We placed an additional 25 emerging adult offspring into $8 \mathrm{~cm} \times 9.5 \mathrm{~cm} \times 6.5 \mathrm{~cm}$ Plexiglass boxes with ventilation holes (provisioned as above). When these bees were 7 days old, they were flashfrozen and stored at $-80{ }^{\circ} \mathrm{C}$ for qPCR analysis (see Section 2.8).

\subsection{Intruder assay}

The intruder assay is a lab-based assay of honey bee aggression. Previous studies show that high aggression in the intruder assay is associated with greater survivorship following pesticide treatment, and lower levels of mite infestation (Rittschof et al. 2015). Thus, this assay provides a behavioral predictor of health resilience (Rittschof et al. 2015). When bees were 7 days old, we moved petri dishes to a temperaturecontrolled ventilated laboratory space $\left(25-30{ }^{\circ} \mathrm{C}\right.$, natural light). We assigned dishes across observers (typically 2 observers per day) and placed the dishes on a table top in random order (observers were blind to treatment identity). We allowed bees to acclimate for $1 \mathrm{~h}$ prior to testing. At the start of the assay, one intruder bee (a forager from a colony that was not otherwise involved in our study) was marked on the thorax with a paint pen (Elmer's, High Point, NC, USA) for identification and introduced into the dish of 4 bees. Over $1 \mathrm{~min}$, we scored five behaviors displayed by group members towards the intruder: (1) antennation, which was movement of the antenna of a group member in contact with the intruder bee. An antennation event ended when the group member turned away from the intruder; (2) antennation with mandibles open, which was similar to antennation, but with the mandibles parted; (3) biting, in which the mandibles of a group member clamped down or pulled on the intruder bee; (4) abdominal flexion, in which a group member mounts or clings to the intruder while flexing its abdomen but not extruding stinger; and (5) stinging, in which a group member mounts or clings to the intruder, flexes her abdomen, and extrudes her stinger. We quantified 
stinging in two ways, the number of discrete stinging attempts and the total time spent stinging regardless of number of attempts (sting duration). Both attempts and duration are reasonable measures of aggression severity. We summed the total tallies for each behavior per dish (reported as an average per bee). We analyzed these tallies as well as a total aggression score per bee. The total aggression score is a composite score that weights individual behaviors for severity (1-5 as per the order specified above). We calculated two different total scores, one incorporating sting attempts and the other incorporating sting duration.

\subsection{Egg protein and size analysis}

We thawed samples $(N=10$ samples, 2 eggs/sample) on ice, added $200 \mu \mathrm{L}$ of distilled water, and homogenized with a micro-pestle (Wegener et al. 2010; Foray et al. 2012). We quantified the protein content of $50 \mu \mathrm{L}$ of the homogenate with a Micro BCA Protein Assay kit following the manufacturer's protocol (Thermo Scientific, Waltham, MA, USA). For a small number of cold stressed and control eggs in a pilot study ( $N=14$ control and $N=19$ cold stressed eggs across two queens), we measured egg size (width and length) from photographs using ImageJ (Schneider et al. 2012). Egg measurement procedures were adapted from Al-Lawati and Bienefeld (2009). We found no treatment differences in egg size (Table SIII), and so we did not carry out size measurements in our main study.

\subsection{Adult immune gene expression analysis}

We used qPCR to measure mRNA expression levels of a panel of immune genes whose expression values reflect parasite and pathogen susceptibility or infection (Evans et al. 2006; Doublet et al. 2017). For the panel, we selected eight genes from a meta-analysis of a 19-transcriptome dataset (Doublet et al. 2017; genes and their functions are described in Table I, primer sequences are listed in Table SIV). We included genes canonically associated with the insect immune system that are also shown to be differentially expressed in association with a wide range of pathogens and stressors in honey bees (including mite feeding and viral, fungal, and bacterial infections; Doublet et al. 2017). We chose genes associated broadly with immune challenge because the specific nature of potential impacts of queen stress on offspring immune function is unknown. We evaluated gene expression in the fat body, an abdominal tissue that functions as a part of the humoral immune system (Wilson-Rich et al. 2008; Richard et al. 2012) and is analogous to the liver and white adipose tissue of vertebrates (Nunes et al. 2013).

We assessed immune gene expression for control and cold stress offspring from 3 queens selected at random. This sub-sampling approach allowed us to assess treatment groups with adequate replication across a range of genotypes, but in a cost-effective manner. Three genotypes are a typical level of replication for this type of molecular approach (Alaux et al. 2009; Naeger et al. 2011). We removed the gut from abdomens partially thawed in RNAlater (Thermo Scientific, Waltham, MA, USA). We extracted RNA from the remaining tissue attached to the abdominal cuticle using E.Z.N.A. HP Total RNA kit, which includes an on-column DNAse treatment (Omega Bio-Tek, Norcross, Georgia, USA). We homogenized tissue in a lysis buffer (included in Total RNA kit) with four 0.7-mm zirconia/silica beads (Bio-spec, Bartlettesville, OK, USA) in a bench top homogenizer (MP Biomedicals, Santa Anna, CA, USA). We quantified the RNA using a CLARIOstar microplate reader with LVis plate (BMG Labtech, Cary, NC, USA), synthesized cDNA using 200 ng RNA and SensiFAST cDNA Synthesis Kit (Bioline, Taunton, MA, USA), and performed qPCR on a Quanta Studio 6 (Thermo Scientific, Waltham, MA, USA) with $10 \mu \mathrm{L}$ reactions (in triplicate) using PerfeCTa SYBR green supermix (Quanta Bio, Beverly, MA, USA). We used previously published primers (see Table SIV) and quantified relative RNA against a DNA standard curve for all genes except apidaecin-1. Due to its short exons, it was not possible to design primers to amplify the standard curve for this target; therefore, we used the delta CT method to assess relative quantity.

Target gene quantities were normalized to the geometric mean of 2 endogenous control genes, gapdh (GB50902) and rp49 (GB47227). We selected these two genes based on pilot assessment 
Table I. Immune panel genes derived from Doublet et al. (2017). Primer sequences and sources are listed in Table SIV

\begin{tabular}{lll}
\hline Gene & NCBI ID & \multicolumn{1}{c}{ Description } \\
\hline abaecin & 406144 & Antimicrobial peptide \\
defensin-1 & 406143 & Antimicrobial peptide \\
hymenoptaecin & 406142 & Antimicrobial peptide \\
prophenoloxidase & 406155 & Melanizing agent \\
prophenoloxidase activator & 726126 & Activator for melanizing agent \\
vitellogenin & 406088 & Storage protein \\
apidaecin & 406115 & Antimicrobial peptide \\
lysozyme-2 & 724899 & Antimicrobial peptide \\
\hline
\end{tabular}

that showed low (less than 20\%) coefficient of variation for each of these genes in the fat body. In our samples, we verified that these two genes had a coefficient of variation across all samples that was less than or equal to $20 \%$, and that these genes were not differentially expressed as a function of treatment.

\subsection{Data analysis}

We collected egg hatching success data for 20 queens across the entire study. We analyzed egg hatching success (yes/no) using McNemar's Test (JMP Pro 13.2), paired for queen. We analyzed egg protein content and behavioral data using linear mixed models (the lmer and glmer functions in the lme4 package in R (Bates et al. 2015; R Core Team 2018). We coded random effects using a random intercept approach (Harrison et al. 2018). For all linear mixed models, we assessed treatment significance using a likelihood ratio test between models with and without the treatment main effect. For egg protein content, we coded cold stress treatment as a fixed effect and queen as a random effect; we excluded round and foster hives as factors from this analysis since eggs were collected and assessed immediately after they were laid, and we collected eggs for only the first two rounds of the experiment ( $N=8$ queens), limiting seasonal variation. We used only the first two rounds in order to improve numbers of surviving individuals for later stages of data collection (development time, behavior, and immune data) in later rounds.
For the analysis of development time and aggressive behavior, we treated queen and foster hive as random effects, with experiment start date and cold stress treatment as fixed effects. Because offspring from some queens failed to hatch, we have data for development time and behavior from $N=11$ total queens. We assessed treatment effects on development time using a proportional hazards survival analysis (JMP Pro 13.2). We found no significant differences across blinded observers in aggression scores, so data were pooled across observers. Total aggression scores (including either sting attempts or duration) were $\log$ transformed for normality prior to analysis with linear mixed models (Li-Byarlay et al. 2014; Rittschof 2017). Singular aggressive behaviors (antennation, antennation with mandibles open, biting, flexion, sting attempts, and sting duration) were assessed using generalized linear mixed models with a Poisson distribution and log link function.

We analyzed gene expression data for a subset of three randomly selected queens to achieve replication across genotypes. This type of approach is typical for gene expression analyses where it is cost prohibitive to replicate sampling as broadly as assessments of other phenotypes (Naeger et al. 2011). Because gene expression patterns were similar among queens, we pooled data from all three prior to analysis. Data distributions varied by gene, and so we used two-tailed $t$ tests when data was normally distributed, and two-tailed Wilcoxon tests for non-normal data (indicated in Section 3). 


\section{RESULTS}

Due to variation in the available offspring within hives, we were only able to collect handling control offspring (see Section 2.4) for 9 queens throughout the entire study. Moreover, these frames generally contained a low number of usable offspring, and so we were unable to confidently statistically analyze handling control data for all phenotypes. However, we found no difference in offspring hatching success between the two controls (Table SV, $P=1.0$ ), and offspring development time and total aggression score (attempts) did not consistently differ (Figs. S23 ). Taken together, we observed minimal consistent differences comparing the handling control with the caged queen control (prior to cold stress, see Section 2.4), and thus limited evidence that handling stress had a distinct impact on offspring phenotype. As a result, for a small number of cases where the control frame failed to produce adequate offspring for analyses (2 queens for the development time and behavioral analyses), we substituted results from the handling control for the control in order to maximize sample sizes and power.

Cold stress significantly decreased egg hatching success relative to the control (Table II, $N=$ 20 queens, McNemar test, $X^{2}{ }_{1}=4, P=0.046$ ), and delayed offspring development by more than half a day on average (Figure $1, N=11$ queens, Mean/S.E.: control 20.6/0.02 days, cold stress 21.3/0.02; proportional hazards $X^{2}{ }_{1}=247.4$, $P<0.0001)$. Offspring from 9 of 11 queens showed a consistent pattern of delayed development following cold stress (Figure 1). Despite these impacts on development, egg protein content did not change with cold stress (Figure 2, $N=$ 8 queens, Mean/S.E.: control 6.4/0.37 $\mu \mathrm{g}$,

Table II. Cold stress significantly decreased offspring egg hatching success

\begin{tabular}{lll}
\hline & Did not hatch & Hatched \\
\hline Control & 3 & 17 \\
Cold stress & 6 & 14 \\
\hline
\end{tabular}

$N=20$ queens/treatment, McNemar's test, $P<0.046$ treatment 6.0/0.25 $\mu \mathrm{g}$; $\mathrm{LMM} X^{2}{ }_{1}=0.60, P=$ $0.44)$, nor did egg size (Table SIII).

Adult phenotypes showed variable impacts of cold stress. Cold stress did not significantly impact total aggression score, a composite measure that weighted behaviors on the basis of severity (antennation, antennation with mandibles open, biting, abdomen flexion, and either sting attempts or duration). Total aggression score showed no treatment effects, whether the composite included either attempts or duration (Figure 3; $N=11$ queens; $\mathrm{LMM}_{\text {attempts }} X^{2}{ }_{1}=0.10, P=0.75$; $\mathrm{LMM}_{\text {duration: }} X^{2}{ }_{1}=0.47, P=0.49$ ). Simpler linear mixed models with only queen and treatment (excluding round and foster hive) gave similar results. However, taking each aggressive behavior independently, cold stress treatment had some effects (Figure 4): it significantly decreased antennation $\left(X^{2}{ }_{1}=8.9, P=0.003\right)$ and significantly increased sting duration $\left(X^{2}{ }_{1}=16.8\right.$, $P<0.0001)$. No other behaviors showed treatment effects (mandibles $X^{2}{ }_{1}=1.01, P=0.31$; bit$\operatorname{ing} X^{2}{ }_{1}=1.05, P=0.31$; flexion $X^{2}{ }_{1}=0.22, P=$ 0.64 ; sting attempts $X^{2}{ }_{1}=0.29, P=0.59$ ). Adult offspring showed no differences in immune gene expression as a function of cold stress (Table SVI), although there is notable variance within treatments (Figure 5).

\section{DISCUSSION}

Transmissive maternal effects are common in disturbed environments, and could have important impacts on individual health and colony resilience in social insects, where one or a small number of queens produce all other colony members. We used an artificial, short-term cold stress, applied to otherwise healthy queens, as a proof-of-concept approach to assess evidence that maternal stress is transmitted to worker offspring. We found that maternal stress decreased the probability of egg hatching and delayed worker development. However, once workers reached adulthood, maternal stress had variable lasting impacts on adult worker behavior and minimal impacts on immune gene expression. This work provides a baseline understanding of the likely impacts of queen stress on worker phenotypes. 


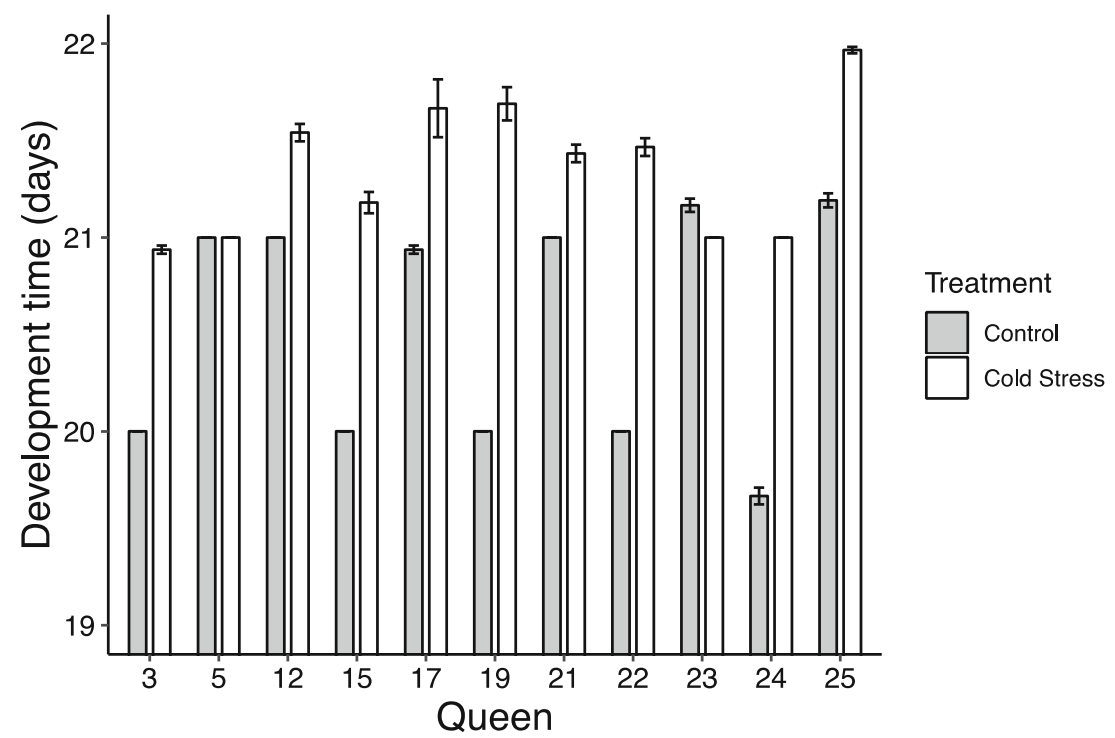

Figure 1. Cold stress significantly delayed worker offspring development time. Offspring laid after queen cold stress took longer to emerge to adulthood compared with control offspring laid by these same queens prior to cold stress. This pattern was consistent for 9 of 11 queens assessed. Data shown is the average ( \pm S.E.) days from oviposition to adult emergence. The numbers along the $x$-axis designate different queens used in the study (queen identity is consistent across all figures). The degree of variance in offspring development time differed across queens, and as a result, error bars are small or absent in some cases. Note: to make data as clear as possible, the $y$-axis on this figure is scaled from 19 to 22 days.

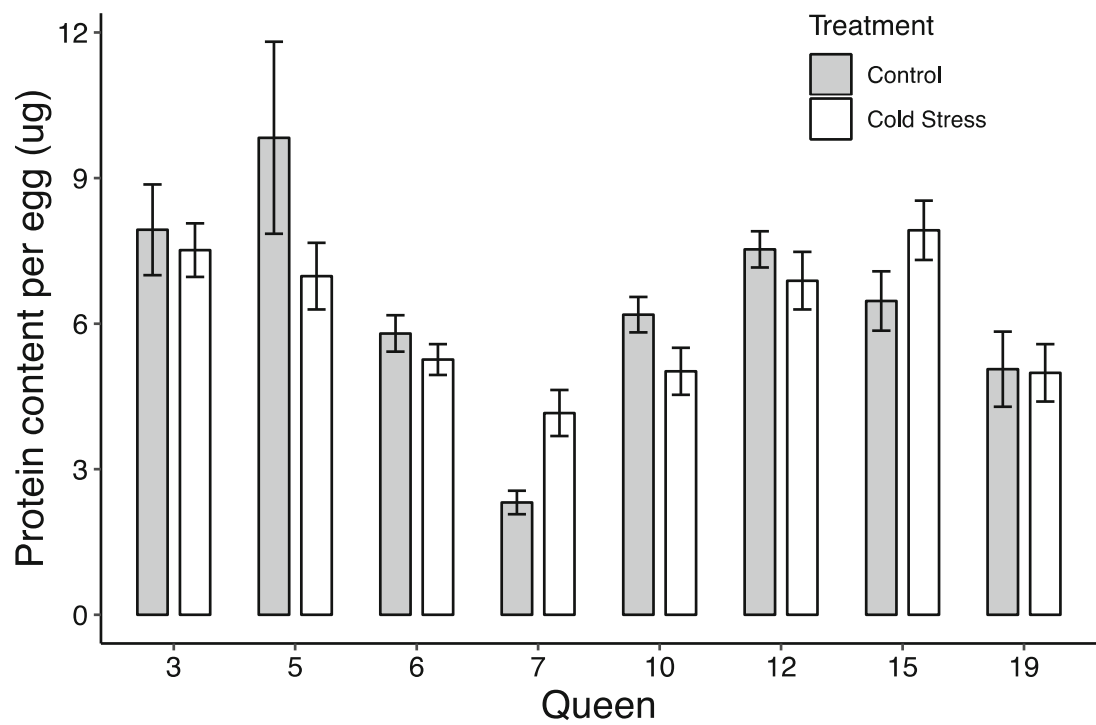

Figure 2. Cold stress did not impact worker offspring egg protein content. Eggs were collected and analyzed from $N=8$ queens from the first two rounds of the experiment. Eggs laid by queens following cold stress showed no significant changes in protein content compared with eggs laid by these same queens prior to cold stress $(P=0.44)$. 


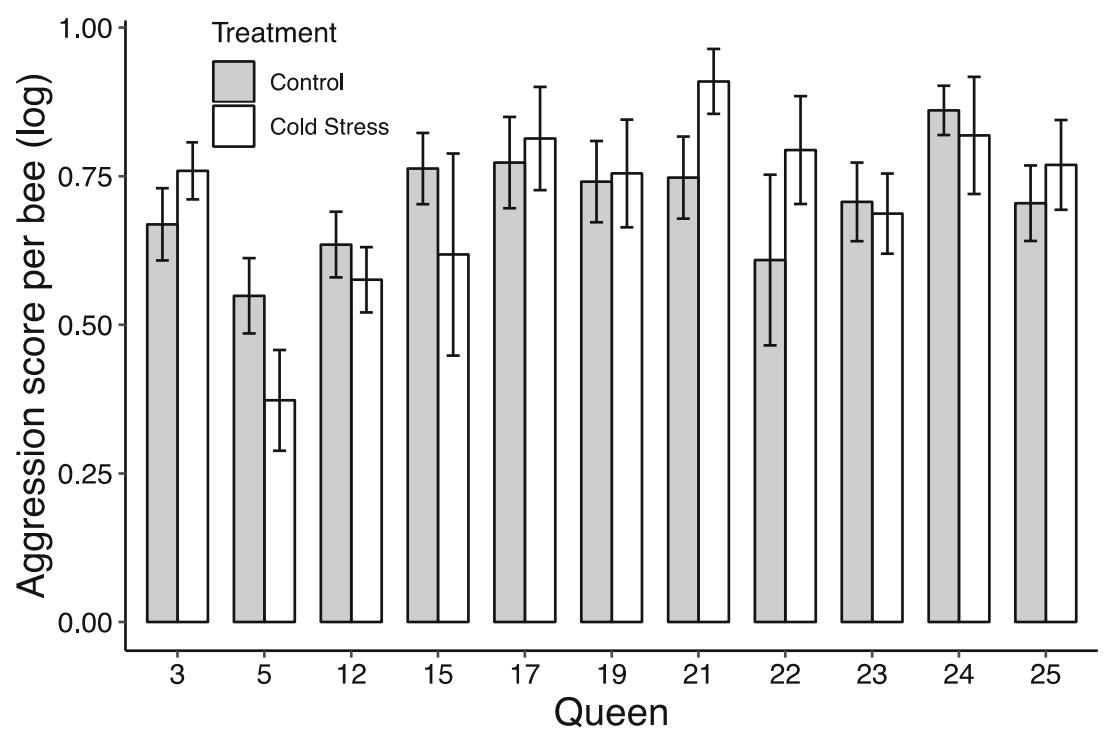

Figure 3. Adult worker total aggression score is not affected by queen cold stress treatment. A composite aggression score incorporating multiple aggressive behaviors did not show significant treatment differences as a function of cold stress to the queen $(P=0.75)$. Data are from offspring laid by a given queen before (control) and after she was exposed to cold stress. Data shown are for the total aggression score calculated with sting attempts. Patterns for total scores using sting duration were very similar (data not shown). Data were log transformed prior to analysis, and bars represent mean $( \pm$ S.E. $)$.

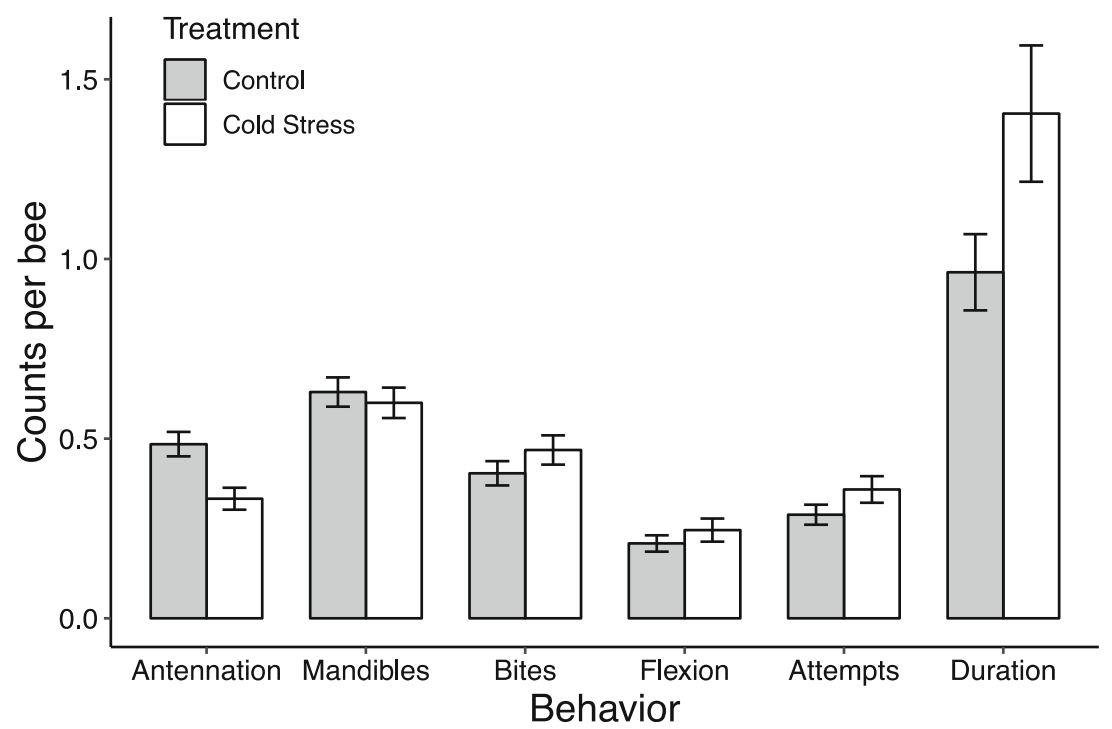

Figure 4. Some aggressive behaviors show significant variation as a function of cold stress. Data analyzed in Figure 3 are presented in terms of the individual behaviors that make up the composite total scores. Bars represent mean $( \pm$ S.E. $)$. Cold stress significantly decreased the frequency of antennation towards the intruder $(P=0.003)$, while it significantly increased the duration workers stung the intruding bee $(P<0.0001)$. No other behaviors showed significant differences as a function of cold stress. 

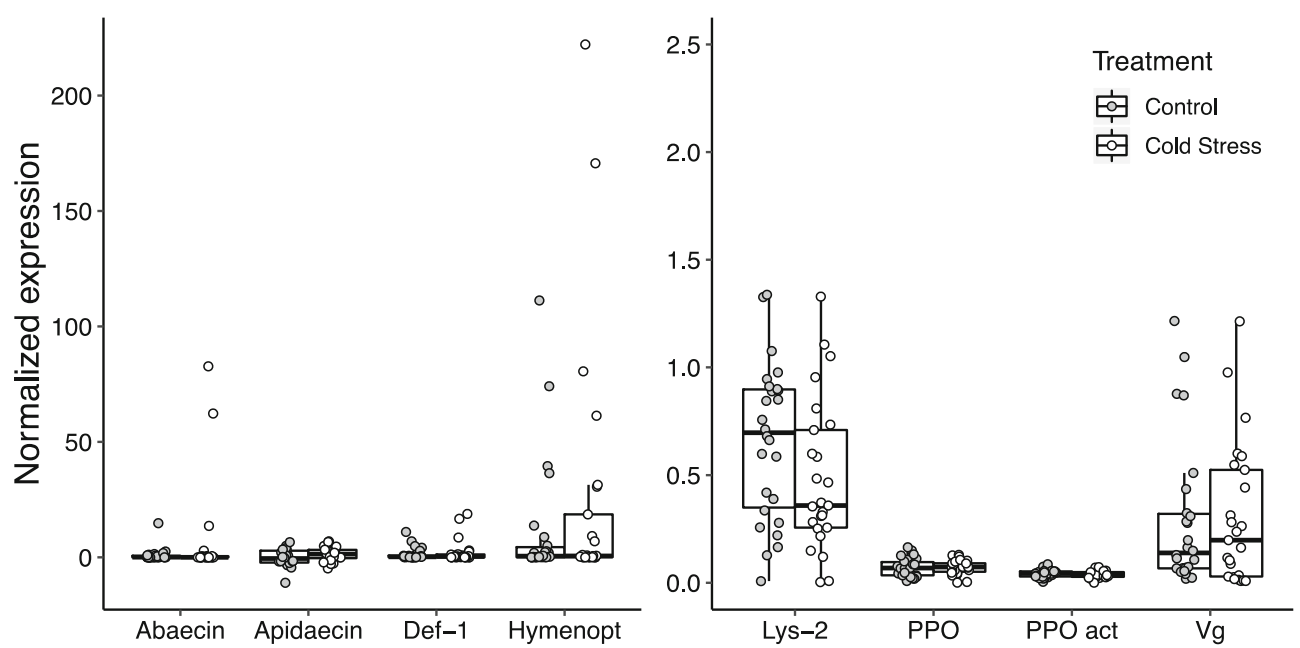

Figure 5. Adult worker immune gene expression was not significantly different following queen cold stress. Data are normalized to the geometric mean of two endogenous control genes. Data are plotted as boxplots, and points represent individual data points (pooled across offspring from three queens). No genes showed significant differences as a function of treatment, although some genes showed substantial variation within treatment groups.

In our study, the strongest impacts of queen cold stress were observed at the egg stage. Pilot data showed that many offspring laid within the first $24 \mathrm{~h}$ following queen cold stress failed to hatch entirely, and the current results, which target offspring laid 24-48 h after cold stress, also show strong evidence of egg hatching failure. Despite evidence that stress impacts protein provisioning to eggs in other insect species (Diss et al. 1996), we found no evidence that cold stress impacts protein provisioning or egg size, although notably, both of these characteristics varied across queens.

The cold stress effects we observed could be a direct result of egg exposure to cold temperatures, as opposed to an indirect effect of queen stress on some feature of egg provisioning or development. Eggs less than $2 \mathrm{~h}$ old have a low survival rate following cold temperature exposure because they are in the pre-cellular, syncytial state (Collins and Mazur 2006); this sensitivity to direct cold exposure may also extend to the late-stage oocytes present at the time of our queen cold stress. The difference in hatching success comparing eggs laid $24 \mathrm{~h}$ and $48 \mathrm{~h}$ following the cold stress suggests eggs closer to oviposition are relatively more sensitive to cold or queen stress. We did not track offspring beyond $48 \mathrm{~h}$ following the queen cold temperature stress, and it is possible that over a longer time frame, egg development and hatching rate would return to normal. Thus, stress to the queen could have a temporary but severe impact on offspring survivorship. Multi-day loss in worker production could have important impacts on colony stability, especially if other stressors simultaneously influence behavior and survivorship of other colony members (Khoury et al. 2011; Goulson et al. 2015; McMahon et al. 2016). Inviable female eggs may make it more difficult for the colony to replace a stressed queen because there are fewer choices among female offspring (Sagili et al. 2018).

In animals that produce offspring over relatively short time windows, asynchrony between maternal stressors and embryo production could protect offspring from certain maternal stress effects. In contrast, in species in which offspring development and production is more or less continuous during adulthood (many invertebrates), ephemeral environmental stressors are more likely to impact at least some offspring. For example, in vertebrates, one well-known context for maternal stress effects involves glucocorticoid (a stress hormone) provisioning to embryos. Similar to the temperature effects observed here, this is a passive process 
wherein a presumably temporary spike in maternal glucocorticoids results in increased hormone quantity in egg yolk or elevated transmission of glucocorticoids to the placenta (Sheriff and Love 2013). However, the stressor is only transmitted if elevated hormone levels occur during a critical window of offspring development. Thus, the relative severity of the impacts of transient anthropogenic stressors may depend on the life history patterns of organisms of concern. Insects, although seasonal, produce eggs over extended periods of time, and recent evidence supports the idea that anthropogenic stressors may be impacting this group more broadly than previously appreciated (Hallmann et al. 2017; Lister and Garcia 2018).

In insects, there is evidence for a variety of maternal effects, most of which manifest early in life, but with potential impacts throughout life. Variation in egg size is a common insect maternal effect that impacts offspring body size and the likelihood of embryo survival (Fox et al. 1997). Egg provisioning (specifically the quantity and ratio of three key macronutrients, protein, lipids, and carbohydrates) also shows maternal effectdriven variation within eggs of the same size (McIntyre and Gooding 2000; Al-Lawati and Bienefeld 2009), and in some cases may be a better predictor of offspring hatching success. There are also examples of non-nutritive egg components that are under maternal influence. For example, transgenerational immune priming (TGIP) refers to a phenomenon where offspring from a mother exposed to a pathogen are prepared via maternal effects to mount an immune response. There is evidence of TGIP in honey bees, in which pathogens bound to the yolk protein vitellogenin accumulate in the egg and decrease offspring susceptibility to the pathogen (Salmela et al. 2015).

Some components of early-life variation, like small size at hatching, can be compensated for during the larval stage with extended development time (Fox and Dingle 1994). In keeping with this pattern, we found that queen cold stress delayed development. The mechanism of this effect is unclear, especially since we found no obvious source of variation that may have been compensated for by delayed development: there was no evidence that egg size or provisioning changed as a function of cold stress. In the honey bee, subtle changes in rearing temperature impact adult brain structure and are known to influence development rate and survivorship; pupae kept at low temperatures throughout development developed more slowly and were less likely to emerge successfully (Groh et al. 2004). Notably, we see developmental delay during a similar time frame in response to even an extremely brief temperature treatment.

Early life stressors can have surprisingly persistent impacts on other nutrition-dependent developmental phenotypes (Pechenik 2006); developmental timing is one such phenotype, mediated by juvenile hormone and endocrine signaling from metabolic tissues including the fat body (Mirth and Riddiford 2007). Thus, it is possible that queen stress had persistent effects on developmental pacing and metabolic physiology. Stress often accelerates aging processes in adult worker bees (Perry et al. 2015), but few studies have addressed how pre-adult stressors impact larval or pupal development rate (Scofield and Mattila 2015; Wang et al. 2016).

We used adult behavioral measures to infer whether maternal cold stress makes worker bees more susceptible to health stressors during adulthood. We assessed variation in aggression, a behavior broadly associated with pesticide tolerance, mite resistance, colony productivity, and overwintering success (Camazine 1986; Wray et al. 2011; Rittschof et al. 2015). Queen cold stress did not cause significant changes in a composite aggression score, which was correlated with health effects in previous studies (Rittschof et al. 2015). Moreover, queen cold stress had opposing effects on two aggression behaviors on opposite ends of the severity spectrum: it decreased the antennation response to invading bees, but increased stinging effort. Such contrasting effects are atypical in previous studies, as changes in low and high level behaviors often covary (Rittschof et al. 2015; Rittschof 2017). It is unclear why queen stress would have these differential effects on aggression-relevant behaviors, but it may suggest that perception of an invading bee and the strength of the aggressive response are decoupled following cold 
stress. In adult worker bees, responsiveness to olfactory aggression cues increases with age (Robinson 1987), driven by underlying nutritional and physiological processes that are rooted in hormone signaling and fat metabolism (Breed et al. 2004). A change in this developmental pacing (established in juvenile development but perhaps persistent into adulthood) could alter one or more aspects of the aggressive response. Future studies could more carefully evaluate how pre-adult developmental pacing impacts adult longevity, behavior, and other physiological components that could impact aggressive response, e.g., olfactory sensitivity and nutritional stores.

Cold stress had no significant impacts on offspring immune function, as measured using mRNA values at baseline. A second approach that targets a slightly different measure of immune function would be to measure gene expression in response to an immune challenge, a measure of immune activation (Di Prisco et al. 2013) which may have yielded different results (although both measures are important predictors of response to disease, Olav Rueppell personal communication). Some of our genes showed highly right-skewed expression patterns (e.g., Abaecin and Hymenoptaecin ), which is characteristic of immune activation due to infection with a parasite or pathogen. Many individuals showing evidence of immune activation were offspring from cold stressed queens, which could suggest these individuals are more sensitive to infections or other stressors that were uncontrolled and thus possibly present in our study. However, in general, we find very little evidence that baseline immune activity differs in adult worker bees as a function of queen cold stress.

In honey bees, environmental stressors, including pesticide and pathogen exposure, and poor nutrition, act synergistically to cause colony death (Goulson et al. 2015). There is strong evidence that multiple stressors converge on similar physiological and immune processes, additively weakening the health of an individual bee (Johnson et al. 2009; Liess et al. 2016; Doublet et al. 2017; Tosi et al. 2017). However, synergy can manifest in a second way in social insect colonies: different single stressors may each impact different members of the worker population, leading to a decline in the total available workforce (Perry et al. 2015). Our study supports the idea that queen stress decreases the total worker population, but may not have lasting impacts on the health and behavior of individual bees. Given the substantial role the queen plays in maintaining population numbers, and the increased rate of queen failure in managed hives, future studies may benefit from including maternal effects as an important source of worker honey bee stress, and in considering stress synergy at both the individual and colony levels.

\section{AUTHORS' CONTRIBUTIONS}

S.R.P. carried out data collection, analysis, and manuscript writing. J.W.H. assisted in experimental setup and data collection. J.H.P. and H.M.C. conducted molecular analyses C.C.R. designed study, performed data analysis, and wrote manuscript.Funding information

This work is supported by the National Institute of Food and Agriculture, U.S. Department of Agriculture Hatch Program under accession number 1012993, by a Foundation for Food and Agricultural Research Pollinator Health Fund (Grant ID: 549049), and by the University of Kentucky Bucks for Brains Summer Research Program.

\section{COMPLIANCE WITH ETHICAL STANDARDS}

Conflict of interest The authors declare that they have no conflict of interest.

Les impacts du stress maternel sur les phénotypes des ouvrières chez l'abeille mellifère effets maternels / changement climatique / échec de la reine / fonction immunitaire / expression des gènes

Der Einfluss von maternalem Stress auf ArbeiterinnenPhänotypen der Honigbiene

Maternale Effekte / Klimawandel / Königin-Versagen / Immunfunktion / Genexpression 


\section{REFERENCES}

Alaux, C., Sinha, S., Hasadsri, L., Hunt, G.J., Guzman-Novoa, E., Degrandi-Hoffman, G., Uribe-Rubio, J.L., Southey, B.R., Rodriguez-Zas, S. \& Robinson, G.E. (2009) Honey bee aggression supports a link between gene regulation and behavioral evolution. Proc. Natl. Acad. Sci. U. S. A. $106,15400-5$.

Alaux, C., Folschweiller, M., McDonnell, C., Beslay, D., Cousin, M., Dussaubat, C., Brunet, J.L. \& Le Conte, Y. (2011) Pathological effects of the microsporidium Nosema ceranae on honey bee queen physiology (Apis mellifera). J. Invertebr. Pathol. 106, 380-5.

Al-Lawati, H. \& Bienefeld, K. (2009) Maternal Age Effects on Embryo Mortality and Juvenile Development of Offspring in the Honey Bee (Hymenoptera: Apidae). Ann. Entomol. Soc. Am. 102, 881-8.

Amdam, G.V. \& Omholt, S.W. (2002) The Regulatory Anatomy of Honeybee Lifespan. J. Theor. Biol. 216, 209-28.

Amiri, E., Strand, M.K., Rueppell, O. \& Tarpy, D.R. (2017) Queen Quality and the Impact of Honey Bee Diseases on Queen Health: Potential for Interactions between Two Major Threats to Colony Health. Insects 8, 48.

Baer, B., Collins, J., Maalaps, K. \& Boer, S.P.A. (2016) Sperm use economy of honeybee (Apis mellifera) queens. Ecol. Evol. 6, 2877-85.

Barron, A.B. (2015) Death of the bee hive: Understanding the failure of an insect society. Curr. Opin. Insect Sci. 10, 45-50.

Bates, B., Maechler, M., Bolker, B. \& Walker, S. (2015) Fitting linear mixed-effects models using lme4. J. Stat. Softw. 67, 1-48.

Bonduriansky, R., Crean, A.J. \& Day, T. (2012) The implications of nongenetic inheritance for evolution in changing environments. Evol. Appl. 5, 192-201.

Breed, M.D., Guzman-Novoa, E. \& Hunt, G.J. (2004) Defensive behavior of honey bees: organization, genetics, and comparisons with other bees. Annu. Rev. Entomol. 49, 271-98.

Camazine, S.M. (1986) Differential reproduction of the mite, Varroa jacobsoni (Mesostigmata: Varroidae), on Africanized and European honey bees (Hymenoptera: Apidae). Ann. Entomol. Soc. Am. 79, 801-3.

Chaimanee, V., Evans, J.D., Chen, Y., Jackson, C. \& Pettis, J.S. (2016) Sperm viability and gene expression in honey bee queens (Apis mellifera) following exposure to the neonicotinoid insecticide imidacloprid and the organophosphate acaricide coumaphos. J. Insect Physiol. $89,1-8$.

Collins, A.M. \& Mazur, P. (2006) Chill sensitivity of honey bee, Apis mellifera, embryos. Cryobiology 53, 22-7.

Crino, O.L., Johnson, E.E., Blickley, J.L., Patricelli, G.L. \& Breuner, C.W. (2013) The effects of experimentally elevated traffic noise on nestling white-crowned sparrow stress physiology, immune function, and life-history. J. Exp. Biol.
DeGrandi-Hoffman, G., Chen, Y. \& Simonds, R. (2013) The Effects of Pesticides on Queen Rearing and Virus Titers in Honey Bees (Apis mellifera L.). Insects 4, 71-89.

Di Prisco, G., Cavaliere, V., Annoscia, D., Varricchio, P., Caprio, E., Nazzi, F., Gargiulo, G. \& Pennacchio, F. (2013) Neonicotinoid clothianidin adversely affects insect immunity and promotes replication of a viral pathogen in honey bees. Proc. Natl. Acad. Sci. U. S. A. 110, 18466-71.

Diss, A.L., J. G. Kunkel, M. E. Montgomery, D. E. Leonard (1996) Effects of maternal nutrition and egg provisioning on parameters of larval hatch, survival and dispersal in the gypsy moth, Lymantria dispar L. Oecologia 106, 470-7.

Doublet, V., Poeschl, Y., Gogol-Doring, A., Alaux, C., Annoscia, D., et al. (2017) Unity in defence: honeybee workers exhibit conserved molecular responses to diverse pathogens. BMC Genomics 18, 207.

Dussaubat, C., Maisonnasse, A., Crauser, D., Tchamitchian, S., Bonnet, M., Cousin, M., Kretzschmar, A., Brunet, J.-L. \& Le Conte, Y. (2016) Combined neonicotinoid pesticide and parasite stress alter honeybee queens' physiology and survival. Sci. Rep. 6, 31430.

Evans, J.D., Aronstein, K., Chen, Y.P., Hetru, C., Imler, J.L., Jiang, H., Kanost, M., Thompson, G.J., Zou, Z. \& Hultmark, D. (2006) Immune pathways and defence mechanisms in honey bees Apis mellifera. Insect Mol. Biol. 15, 645-56.

Foray, V., Pelisson, P.-F., Bel-Venner, M.-C., Desouhant, E., Venner, S., Menu, F., Giron, D. \& Rey, B. (2012) A handbook for uncovering the complete energetic budget in insects: the van Handel's method (1985) revisited. Physiol. Entomol. 37, 295-302.

Forcada, J. \& Hoffman, J.I. (2014) Climate change selects for heterozygosity in a declining fur seal population. Nature 511, 462.

Fox, C.W. \& Dingle, H. (1994) Dietary mediation of maternal age effects on offspring performance in a seed beetle (Coleoptera: Bruchidae). Funct. Ecol. 8, 600-6.

Fox, C.W., Thakar, M.S. \& Mousseau, T.A. (1997) Egg size plasticity in a seed beetle: an adaptive maternal effect. Am. Nat. 149, 149-63.

Gibbs, M., Breuker, C.J., Hesketh, H., Hails, R.S. \& Van Dyck, H. (2010) Maternal effects, flight versus fecundity trade-offs, and offspring immune defence in the Speckled Wood butterfly, Pararge aegeria. BMC Evol. Biol. 10, 345 .

Goulson, D., Nicholls, E., Botias, C. \& Rotheray, E.L. (2015) Bee declines driven by combined stress from parasites, pesticides, and lack of flowers. Science $\mathbf{3 4 7}$, 1255957.

Graham, J.M. (2015) The Hive and the Honey Bee. Dadant \& Sons, Hamilton, Illinois.

Groh, C., Tautz, J. \& Rossler, W. (2004) Synaptic organization in the adult honey bee brain is influenced by brood-temperature control during pupal development. Proc Natl. Acad. Sci. U. S. A. 101, 4268-73. 
Hallmann, C.A., Sorg, M., Jongejans, E., Siepel, H., Hofland, N., et al. (2017) More than 75 percent decline over 27 years in total flying insect biomass in protected areas. PLoS One 12, e0185809.

Harrison, X.A., Donaldson, L., Correa-Cano, M.E., Evans, J., Fisher, D.N., Goodwin, C.E.D., Robinson, B.S., Hodgson, D.J. \& Inger, R. (2018) A brief introduction to mixed effects modelling and multi-model inference in ecology. PeerJ 6, e4794.

Jeffs, C.T. \& Leather, S.R. (2014) Effects of extreme, fluctuating temperature events on life history traits of the grain aphid, Sitobion avenae. Entomol. Exp. Appl. 150, 240-9.

Johnson, R.M. (2015) Honey Bee Toxicology. Annu. Rev. Entomol. 60, 415-34.

Johnson, R.M., Pollock, H.S. \& Berenbaum, M.R. (2009) Synergistic interactions between in-hive miticides in Apis mellifera. J. Econ. Entomol. 102, 474-9.

Keiser, C.N. \& Mondor, E.B. (2013) Transgenerational Behavioral Plasticity in a Parthenogenetic Insect in Response to Increased Predation Risk. J. Insect Behav. 26, 603-13.

Khoury, D.S., Myerscough, M.R. \& Barron, A.B. (2011) A quantitative model of honey bee colony population dynamics. PLoS One 6, e18491.

Klein, S., Cabirol, A., Devaud, J.-M., Barron, A.B. \& Lihoreau, M. (2017) Why Bees Are So Vulnerable to Environmental Stressors. Trends Ecol. Evol. 32 , 26878.

Lacour, G., Vernichon, F., Cadilhac, N., Boyer, S., Lagneau, C. \& Hance, T. (2014) When mothers anticipate: Effects of the prediapause stage on embryo development time and of maternal photoperiod on eggs of a temperate and a tropical strains of Aedes albopictus (Diptera: Culicidae). J. Insect Physiol. 71, 87-96.

Li-Byarlay, H., Rittschof, C.C., Massey, J.H., Pittendrigh, B.R. \& Robinson, G.E. (2014) Socially responsive effects of brain oxidative metabolism on aggression. Proc. Natl. Acad. Sci. U. S. A. 111, 12533-7.

Liess, M., Foit, K., Knillmann, S., Schafer, R.B. \& Liess, H.D. (2016) Predicting the synergy of multiple stress effects. Sci. Rep. 6, 32965.

Lister, B.C. \& Garcia, A. (2018) Climate-driven declines in arthropod abundance restructure a rainforest food web. Proc. Natl. Acad. Sci. U. S. A. 115, E10397-E406.

Mackensen, O. (1951) Viability and sex determination in the honey bee (Apis mellifera L .). Genetics 36, 500-9.

Manley, R., Boots, M. \& Wilfert, L. (2015) REVIEW: Emerging viral disease risk to pollinating insects: ecological, evolutionary and anthropogenic factors. J. Appl. Ecol. 52 , 331-40.

Marshall, D.J. \& Uller, T. (2007) When is a maternal effect adaptive? Oikos 116, 1957-63.

McIntyre, G.S. \& Gooding, R.H. (2000) Egg size, contents, and quality: maternal-age and -size effects on house fly eggs. Can. J. Zool. 78, 1544-51.
McMahon, D.P., Natsopoulou, M.E., Doublet, V., Furst, M., Weging, S., Brown, M.J., Gogol-Doring, A. \& Paxton, R.J. (2016) Elevated virulence of an emerging viral genotype as a driver of honeybee loss. Proc. Biol. Sci. 283.

McMenamin, A.J. \& Genersch, E. (2015) Honey bee colony losses and associated viruses. Curr. Opin. Insect Sci. 8 .

Mirth, C.K. \& Riddiford, L.M. (2007) Size assessment and growth control: how adult size is determined in insects. Bioessays 29, 344-55.

Naeger, N.L., Van Nest, B.N., Johnson, J.N., Boyd, S.D., Southey, B.R., Rodriguez-Zas, S.L., Moore, D. \& Robinson, G.E. (2011) Neurogenomic signatures of spatiotemporal memories in time-trained forager honey bees. J. Exp. Biol. 214, 979-87.

Nunes, F.M., Ihle, K.E., Mutti, N.S., Simoes, Z.L.P. \& Amdam, G.V. (2013) The gene vitellogenin affects microRNA regulation in honey bee (Apis mellifera) fat body and brain. J. Exp. Biol. 216, 3724-32.

Pechenik, J.A. (2006) Larval experience and latent effectsmetamorphosis is not a new beginning. Integr. Comp. Biol. 46, 323-33.

Perez-Sato, J.A., Kärcher, M.H., Hughes, W.O.H. \& Ratnieks, F.L.W. (2015) Direct introduction of mated and virgin queens using smoke: a method that gives almost $100 \%$ acceptance when hives have been queenless for 2 days or more. J. Apic. Res. 47, 24350.

Perry, C.J., Sovik, E., Myerscough, M.R. \& Barron, A.B. (2015) Rapid behavioral maturation accelerates failure of stressed honey bee colonies. Proc. Natl. Acad. Sci. U. S. A. $112,3427-32$.

Pettis, J.S., Rice, N., Joselow, K., vanEngelsdorp, D. \& Chaimanee, V. (2016) Colony Failure Linked to Low Sperm Viability in Honey Bee (Apis mellifera) Queens and an Exploration of Potential Causative Factors. PLoS One 11 , e0147220.

Räsänen, K. \& Kruuk, L.E. (2007) Maternal effects and evolution at ecological time-scales. Funct. Ecol. 21, 408-21.

Richard, F.-J., Holt, H.L. \& Grozinger, C.M. (2012) Effects of immunostimulation on social behavior, chemical communication and genome-wide gene expression in honey bee workers (Apis mellifera). BMC Genomics 13,558 .

Rinderer, T.E. (2008) Bee Genetics and Breeding . Northern Bee Books.

Rittschof, C.C. (2017) Sequential social experiences interact to modulate aggression but not brain gene expression in the honey bee (Apis mellifera). Front. Zool. 14, $1-10$.

Rittschof, C.C. \& Robinson, G.E. (2013) Manipulation of colony environment modulates honey bee aggression and brain gene expression. Genes Brain Behav. 12, 802-11.

Rittschof, C.C., Coombs, C.B., Frazier, M., Grozinger, C.M. \& Robinson, G.E. (2015) Early-life experience 
affects honey bee aggression and resilience to immune challenge. Sci. Rep. 5, 15572.

Rittschof, C.C., Vekaria, H.J., Palmer, J.H. \& Sullivan, P.G. (2018) Brain mitochondrial bioenergetics change with rapid and prolonged shifts in aggression in the honey bee, Apis mellifera. J. Exp. Biol. 221.

Robinson, G.E. (1987) Modulation of alarm pheromone perception in the honey bee: evidence for division of labor based on hormonally regulated response thresholds. J. Comp. Physiol. A Sens. Neural Behav. Physiol. 160, 613-9.

Sagili, R.R., Metz, B.N., Lucas, H.M., Chakrabarti, P. \& Breece, C.R. (2018) Honey bees consider larval nutritional status rather than genetic relatedness when selecting larvae for emergency queen rearing. Sci. Rep. 8, 7679.

Salmela, H., Amdam, G.V. \& Freitak, D. (2015) Transfer of Immunity from Mother to Offspring Is Mediated via Egg-Yolk Protein Vitellogenin. PLoS Pathog. 11, e1005015.

Schneider, C.A., Rasband, W.S. \& Eliceiri, K.W. (2012) $\mathrm{NIH}$ image to imageJ: 25 years of image analysis. Nat. Methods 9, 671-5.

Scofield, H.N. \& Mattila, H.R. (2015) Honey bee workers that are pollen stressed as larvae become poor foragers and waggle dancers as adults. PLoS One 10, $\mathrm{e} 0121731$.

Sheriff, M.J. \& Love, O.P. (2013) Determining the adaptive potential of maternal stress. Ecol. Lett. 16, 271-80.

Smith, K.M., Loh, E.H., Rostal, M.K., Zambrana-Torrelio, C.M., Mendiola, L. \& Daszak, P. (2013) Pathogens, pests, and economics: drivers of honey bee colony declines and losses. Ecohealth 10, 434-45.

Strasser, E.H. \& Heath, J.A. (2013) Reproductive failure of a human-tolerant species, the American kestrel, is associated with stress and human disturbance. J. Appl. Ecol. 50, 912-9.

Sulmon, C., van Baaren, J., Cabello-Hurtado, F., Gouesbet, G., Hennion, F., et al. (2015) Abiotic stressors and stress responses: What commonalities appear between species across biological organization levels? Environ. Pollut. 202, 66-77.

Tarpy, D.R., Keller, J.J., Caren, J.R. \& Delaney, D.A. (2012) Assessing the Mating 'Health' of Commercial Honey Bee Queens. J. Econ. Entomol. 105, 20-5.
Team, R.C. (2018) R: A language and environment for statistical computing. R Foundation for Statistical Computing, Vienna.

Tosi, S., Nieh, J.C., Sgolastra, F., Cabbri, R. \& Medrzycki, P. (2017) Neonicotinoid pesticides and nutritional stress synergistically reduce survival in honey bees. Proc. Biol. Sci. 284.

Traynor, K.S., Wang, Y., Brent, C.S., Amdam, G.V. \& Page, R.E. (2017) Young and old honeybee (Apis mellifera) larvae differentially prime the developmental maturation of their caregivers. Anim. Behav. 124, 193-202.

Walton, A. \& Toth, A.L. (2016) Variation in individual worker honey bee behavior shows hallmarks of personality. Behav. Ecol. Sociobiol. 70, 999-1010.

Wang, Y., Kaftanoglu, O., Brent, C.S., Page, R.E. \& Amdam, G.V. (2016) Starvation stress during larval development facilitates an adaptive response in adult worker honey bees (Apis mellifera L.). J. Exp. Biol. 219, 949 .

Wegener, J., Lorenz, M.W. \& Bienefeld, K. (2010) Differences between queen- and worker-laid male eggs of the honey bee (Apis mellifera). Apidologie 41, 116-26.

Wilson-Rich, N., Dres, S.T. \& Starks, P.T. (2008) The ontogeny of immunity: Development of innate immune strength in the honey bee (Apis mellifera). J. Insect Physiol. 54, 1392-9.

Winston, M.L. (1987) The Biology of the Honey Bee. In: (p. 47. Harvard University Press.

Wolf, J.B. \& Wade, M.J. (2009) What are maternal effects (and what are they not)? Philos. Trans. R. Soc. B 364, 1107-15.

Wray, M.K., Mattila, H.R. \& Seeley, T.D. (2011) Collective personalities in honeybee colonies are linked to colony fitness. Anim. Behav. 81, 559-68.

Yu, R. \& Omholt, S.W. (1999) Early developmental processes in the fertilised honeybee (Apis mellifera) oocyte. J. Insect Physiol. 45, 763-7.

Publisher's note Springer Nature remains neutral with regard to jurisdictional claims in published maps and institutional affiliations. 